\title{
Partial Reconstitution of Cutaneous Microvessels in Long-Term Survivors after Allogeneic Bone Marrow Transplantation
}

\author{
P. Haeusermann ${ }^{a} \quad$ E. Kump ${ }^{a, b} \quad$ A. Rovóc ${ }^{c}$ A. Tichellic P. Itin ${ }^{a} \quad$ A. Gratwohl ${ }^{c}$ \\ B.C. Biedermann ${ }^{b, d}$
}

Departments of a Dermatology and ${ }^{b}$ Biomedicine, and c Division of Hematology, Department of Medicine, University Hospital Basel, Basel, and d University Department of Medicine, Kantonsspital Bruderholz, Bruderholz, Switzerland

\section{Key Words}

Graft-versus-host disease $\cdot$ Allogeneic hematopoietic stem

cell transplantation $\cdot$ Long-term survivors

\begin{abstract}
Background: Graft-versus-host disease (GVHD) is a major complication after allogeneic hematopoietic stem cell transplantation (HSCT) and skin is involved in acute and chronic disease. Immune-mediated vessel attack and subsequent microvessel loss have been observed in skin of patients with chronic GVHD. Objectives: To test whether long-term survivors (LTS) after allogeneic HSCT without cutaneous GVHD show signs of persistent vascular remodeling. Methods: Microvessels in skin biopsies were investigated in a cohort of 32 LTS with a median follow-up of 17 years (range 11-26). Five were currently classified as having chronic GVHD other than skin involvement. Results: LTS showed no significant difference in median microvessel density and relative vessel size distribution pattern compared to healthy controls. Past experience of GVHD and current status of chronic GVHD other than skin involvement had no impact on vessel density. In contrast, recipients with chronic cutaneous GVHD of sclerotic type and patients with lichen sclerosus have significant microvessel loss in the upper dermis. Conclusion: The complex therapy of allogeneic HSCT had no sustained effect on
\end{abstract}

\section{KARGER}

Fax +4161306 1234 E-Mail karger@karger.ch www.karger.com

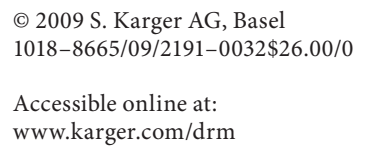

the microvascular architecture of LTS when clinicopathological evidence of cutaneous GVHD is absent. Microvascular remodeling as observed during chronic GVHD recovers completely after resolution of chronic cutaneous GVHD.

Copyright $\odot 2009$ S. Karger AG, Basel

\section{Introduction}

Graft-versus-host disease (GVHD) is a major complication of allogeneic hematopoietic stem cell transplantation (HSCT). Its acute or chronic form is the main cause of nonrelapse morbidity and mortality both as a direct complication (e.g. bronchiolitis obliterans) or via associated immunodeficiency and susceptibility to severe infections [1-3].

Contrary to some expectations, the incidence of GVHD did not significantly decrease over the last 20 years, and still develops in about half of all patients undergoing allogeneic HSCT [4]. While novel transplantation techniques such as reduced-intensity conditioning regimens are associated with less severe acute GVHD rel-

P. Haeusermann and E. Kump contributed equally to this work.

Dr. Peter Haeusermann, MD

Department of Dermatology, University Hospital Basel

Petersgraben 4

$\mathrm{CH}-4031$ Basel (Switzerland)

Tel. +41 6126525 25, Fax +41 6126548 85, E-Mail phaeusermann@uhbs.ch 
ative to myeloablative regimens, the incidence of acute GVHD has remained unchanged $[4,5]$. By comparison, extended eligibility criteria for patients undergoing transplantation (e.g. increased upper age limit), increased human leukocyte antigen disparity, prolonged initial survival due to reduction of early mortality as a consequence of better prophylaxis and treatment strategies of immediate complications of transplantation (e.g. acute GVHD and infections), and increased use of peripheral blood stem cells have increased the risk for chronic GVHD [6].

Cutaneous GVHD is divided into an acute and chronic form and has recently been reviewed by our group [7]. Acute GVHD is pathophysiologically well understood and initiation and maintenance involves various host and recipient immunocompetent cells as well as noncellular molecules $[8,9]$. In contrast, the pathogenesis of chronic GVHD is more complex and thought to be primarily an immune-mediated process that mostly involves alloreactive $\mathrm{CD} 4+$ and $\mathrm{CD} 8+\mathrm{T}$ cells as main effectors [10]. As a hallmark feature, chronic GVHD may present with clinical and laboratory findings that resemble various autoimmune disorders, e.g. bronchiolitis obliterans, Sjögren's syndrome, immune cytopenias, and cutaneous sclerosis, suggesting that dysfunctional humoral immunity might be involved in the pathogenesis as well [11-13].

It has previously been shown that patients with active chronic GVHD have significantly reduced microvessels in skin compared to healthy controls [14]. It has been hypothesized that antiendothelial immune attack and subsequent vessel loss in these patients is the result of alloreactive antiendothelial cytotoxic T cells. However, in this former study we had no access to skin biopsies from longterm survivors (LTS) free of cutaneous GVHD. Therefore, we could not rule out that the process of allogeneic HSCT itself would affect microvascular remodeling in the skin.

In this present study, we investigated skin biopsies of a homogeneous population of 32 recipients more than 10 years after allogeneic HSCT who lacked any clinical signs of cutaneous GVHD at the time of biopsy. The main aim of our investigations was to examine the cutaneous microvasculature in LTS of allogeneic HSCT, to determine whether in these individuals vascular remodeling (vessel size distribution pattern) or restitutio ad integrum (complete reconstitution of vessel quantity and normal vessel size distribution pattern) of the cutaneous microvascular network occurs, and to eventually establish microvascular criteria for subclinical GVHD.

\section{Patients and Methods}

\section{Patients}

We investigated skin biopsies of 32 allogeneic stem cell transplant recipients more than 10 years after myeloablative bone marrow transplantation. Table 1 summarizes the key data of the study population (LTS). Roughly $2 / 3$ of our LTS previously experienced acute GVHD demonstrating classical erythematous rash that resolved within days to a few weeks after standard treatment (systemic glucocorticoids). LTS who previously experienced limited chronic GVHD had localized sclerodermatous skin disease, while patients with extensive chronic GVHD had a generalized cutaneous involvement including fibrosing forms. Our study was a cooperative project related to the single-center prospective study in Basel entitled 'Altered immunity in longterm survivors after allogeneic hematopoietic stem cell transplantation as compared with their respective donors'. All 44 recipients and their respective donors gave written informed consent to the protocol that was approved by the local institutional ethics review board. For the cooperative project, the ethics review board only allowed to take skin biopsies from recipients but not from their respective donors. Of the 44 recipients, 4 refused skin biopsy and the remaining 40 patients gave written informed consent. Of those, 32 samples were considered representative and could be further investigated [ 32 for confocal microscopy and 28 for immunohistochemistry (IHC)]. Eight samples (for confocal microscopy) and 12 samples (for IHC) could not be processed for various technical reasons and were independently qualified as insufficient by two of us (P.H., E.K.). Formalinfixed, paraffin-embedded 6-mm punch biopsies were taken from normal-appearing and sun-protected skin, such as on the back, thigh, abdomen, upper arm or gluteal. At the time of biopsy, all recipients of our cohort did not show any clinical signs of cutaneous GVHD.

Table 1 summarizes all important data of the other investigated groups apart from LTS, i.e. patients with chronic sclerotic GVHD and those who never had allogeneic HSCT (healthy controls, patients with lichen sclerosus, and patients with morphea). Except for healthy controls, 4-mm punch biopsies of the lateral borders of lesional skin were taken exclusively for diagnostic purposes after patient's consent and before the start of this prospective study. All patients with chronic cutaneous GVHD of sclerotic type presented homogeneously with hallmark clinical and histopathological features of chronic sclerotic GVHD of skin. Patients with morphea and lichen sclerosus presented the classical clinical and histopathological characteristics of the respective disease. Normal-appearing skin of healthy controls was taken after patient's consent from the edges of spindle excisions for cosmetically distracting and required removals of benign fibromas and papillomatous melanocytic nevi.

\section{Slide Preparation}

We embedded formalin-fixed skin biopsy samples in paraffin and cut 5 -mm-thick sections. Paraffin was removed by xylol and treatment with 100, 96, and 70\% ethanol. After rinsing the slides in phosphate-buffered saline (PBS; pH 7.4), we demasked the antigens in $10 \mathrm{mmol} / \mathrm{l}$ citrate buffer ( $\mathrm{pH} 6)$ in a steam presser chamber for $2 \mathrm{~min}$. The slides were washed again with PBS. 
Table 1. Key data of all investigated groups

\begin{tabular}{|c|c|c|c|}
\hline & LTS & Chronic sclerotic GVHD & \\
\hline Number of patients & 32 & 5 & \\
\hline $\operatorname{Sex}(m / f)$ & $19(59) / 13(41)$ & $5(100) / 0$ & \\
\hline Median age at transplantation, years & $25(5-50)$ & $47.5(39-56)$ & \\
\hline Median time interval after HSCT, years & $17(11-26)$ & $2(1-5)$ & \\
\hline \multicolumn{4}{|l|}{ Skin status at follow-up examination } \\
\hline Clinical assessment & normal $100 \%$ & sclerodermoid 100\% & \\
\hline Histological assessment & normal $100 \%$ & sclerodermoid 100\% & \\
\hline \multicolumn{4}{|l|}{ Diagnosis } \\
\hline Aplastic anemia & $4(12)$ & - & \\
\hline Acute myeloid leukemia & $12(38)$ & $1(20)$ & \\
\hline Chronic myeloid leukemia & $9(28)$ & - & \\
\hline Acute lymphoblastic leukemia & $4(13)$ & $2(40)$ & \\
\hline Non-Hodgkin lymphoma & $2(6)$ & - & \\
\hline Chronic lymphocytic leukemia & $1(3)$ & $2(40)$ & \\
\hline Myelodysplastic syndrome & $1(3)$ & - & \\
\hline Total body irradiation (yes/no) & $27(84) / 5(16)$ & $5(100)$ & \\
\hline Source of stem cells: bone marrow & $100 \%$ & $100 \%$ & \\
\hline Median nucleated cell dose for transplantation $\left(\times 10^{8} / \mathrm{kg}\right)$ & $3.77(0.59-16.6)$ & $6.43(3.58-13.75)$ & \\
\hline Donor type: matched sibling & $100 \%$ & $100 \%$ & \\
\hline \multicolumn{4}{|l|}{ Previous experience of acute GVHD } \\
\hline None or grade I/grade II-IV & $15(47) / 17(53)^{1}$ & $1(20) / 4(80)$ & \\
\hline \multicolumn{4}{|l|}{ Previous experience of chronic GVHD } \\
\hline None/yes, limited/extensive & $17(53) / 15(47), 10 / 5$ & $3(60) / 2(40)$ & \\
\hline \multicolumn{4}{|l|}{ Current status of chronic GVHD } \\
\hline \multirow[t]{2}{*}{ None/limited/extensive } & $27(84.5) / 2(6) / 3(9.5)$ & n.a. & \\
\hline & Healthy controls & Morphea & Lichen sclerosus \\
\hline Number of samples & 6 & 5 & 5 \\
\hline \multicolumn{4}{|l|}{ Skin status } \\
\hline Clinical assessment & normal skin $100 \%$ & morphea $100 \%$ & lichen scl. $100 \%$ \\
\hline Histological assessment & normal skin $100 \%$ & morphea $100 \%$ & lichen scl. $100 \%$ \\
\hline $\operatorname{Sex}(m / f)$ & $4(67) / 2(33)$ & $2(40) / 3(60)$ & $1(20) / 4(80)$ \\
\hline Median age, years & $46(26-76)$ & $43(15-70)$ & $59(14-68)$ \\
\hline
\end{tabular}

Figures in parentheses indicate ranges or percentages. ${ }^{1}$ Acute GVHD grade 0: $\mathrm{n}=11(34.4 \%)$; grade I: $\mathrm{n}=4(12.5 \%)$; grade II: $\mathrm{n}=$ 13 (40.6\%); grade III: $\mathrm{n}=4(12.5 \%)$; grade IV: $\mathrm{n}=0(0 \%)$.

\section{Immunohistochemistry}

Before IHC, slides were preincubated with $3 \%$ hydrogen peroxide in 50\% methanol. Vascular endothelial cells were detected with antibody against von Willebrand factor (vWF). We incubated slides with rabbit anti-vWF (1:600, DAKO) and a peroxidase-conjugated goat anti-rabbit antibody (EnVision System HRP rabbit, DAKO). DAB EnVision kit was used for detection (DAKO). Slides were analyzed with a light microscope (Leica DM 2500, Leica Microsystems, Wetzlar, Germany) and photographed with a digital camera.

\section{Immunofluorescence}

Nonspecific binding sites were blocked with 3\% fat-free dry milk in PBS for 30 min at room temperature. Vascular endothelial cells were detected with anti-vWF antibody (1:200, DAKO) for
$1 \mathrm{~h}$ at room temperature. Slides were rinsed with PBS and incubated with cyanine 5 (Cy5)-conjugated donkey anti-rabbit antibody (1:800, Jackson ImmunoResearch, West Grove, Pa., USA) for $30 \mathrm{~min}$ at room temperature. The slides were then rinsed again with PBS and mounted under glass with hydrophilic embedding medium (Faramount, DAKO). Immunofluorescence (IF) was detected with a confocal microscope (Zeiss, Jena, Germany).

Quantitative Morphometric Analysis of Microvessel Density

For each patient, one microscopy slide containing a representative paraffin section from the skin biopsy was analyzed. For IHC slides, one image per slide was taken. In the superficial dermis (a subepidermal region 150-200 $\mathrm{mm}$ deep), we determined the area to be analyzed and then counted the number of vessels and measured the diameter of vascular profiles lined by endothe- 

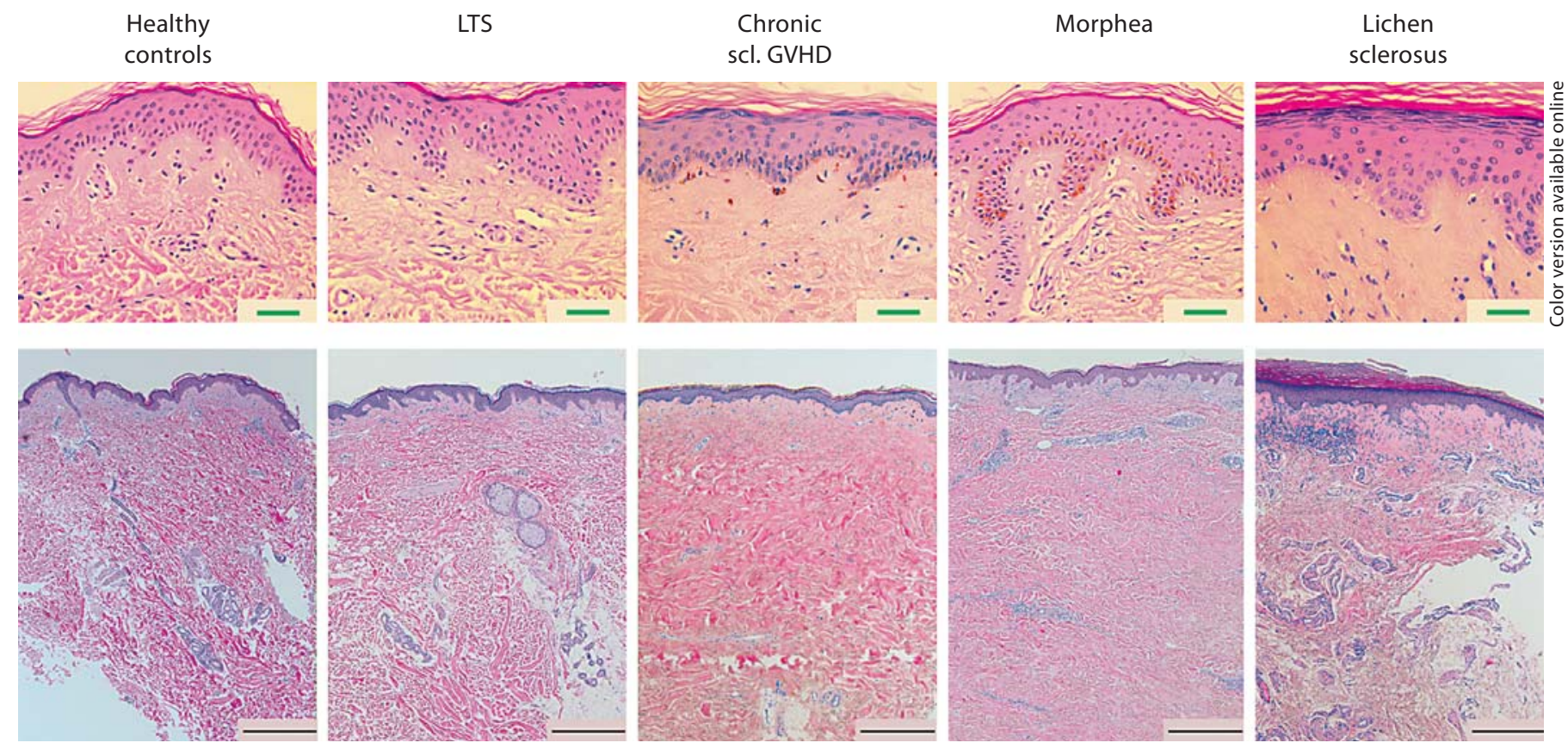

Fig. 1. Typical skin morphology of healthy controls and the four patient groups. Columns show all investigated patient groups and controls. Two different magnifications of hematoxylin/eosinstained paraffin sections are shown for each group. Upper row: superficial dermis, high-power view; lower row: deep dermis, low-power view. Morphological findings are described in the text. Bars $=50 \mu \mathrm{m}$ (upper row) and $500 \mu \mathrm{m}$ (lower row). lial cells using image analysis software $\left(\mathrm{Cell}^{\mathrm{P}}\right.$, Olympus Soft Imaging Solutions, Volketswil, Switzerland). On average, $0.33 \mathrm{~mm}^{2}$ of the superficial dermis were examined per patient. Vessel profiles were categorized according to their diameter (no lumen $/ 0-10$ $\mathrm{mm} / 10-20 \mathrm{~mm} />20 \mathrm{~mm}$ ). Total profile counts $/ \mathrm{mm}^{2}$ as well as profile count per category were determined. Results were calculated as median profile number $/ \mathrm{mm}^{2}$ (total profile density) and median profile number per category divided by median total count (percent of total count). For IF-stained slides, four representative images were taken in the superficial dermis and the median of four results was calculated as representative value per slide. Fluorescence images were analyzed with Adobe ${ }^{\circledR}$ Photoshop ${ }^{\circledR}$ software. Total pixel number of the area to be analyzed was determined. Within this area, the number of pixels matching the wavelength of Cy5 (fig. 1, blue in the online version) was determined. Results were normalized according to the total pixel number of the corresponding area, and were calculated as median pixel number per total area pixel number for each patient group.

\section{Antibodies}

Rabbit anti-vWF (DAKO A0082) was used for vWF detection in both IHC and IF. Donkey anti-rabbit Cy5 (Jackson 711-175152) was used as a secondary antibody in IF. Rabbit serum was used for negative controls in both applications.

\section{Data Analysis and Statistics}

Total profile number $/ \mathrm{mm}^{2}$ as well as relative vWF pixel counts are represented as medians. Mann-Whitney $U$ test was performed for statistical significance to determine differences between pa- tient groups and controls. Differences with $p<0.05$ were considered significant. Comparison between the two endothelial cell measurement methods (profile number $/ \mathrm{mm}^{2}$ and Cy5 matching pixels per total pixels) was analyzed with the Spearman correlation coefficient. Profile size distribution analysis (group comparisons) was tested for statistical significance with a $\chi^{2}$ test. Differences with $\mathrm{p}<0.05$ were considered significant.

\section{Results}

\section{Chronic Sclerotic GVHD and Lichen Sclerosus Are \\ Vanishing Vessel Diseases of the Upper Dermis}

In this study, we investigated the status of microvasculature in skin of a large homogeneous population of allogeneic stem cell recipients with a median follow-up of 17 years after transplantation. We morphometrically assessed number and size categories of blood vessels defined by immunohistochemical positivity for vWF within the upper dermal layers (150-200 mm downward from basal membrane) anatomically corresponding to the compartment of the superficial vascular plexus in skin. Figure 1 shows one representative sample of all investigated disease groups, i.e. LTS $(n=32)$, chronic sclerotic GVHD $(n=5)$, morphea $(n=5)$, lichen sclerosus $(n=5)$ 


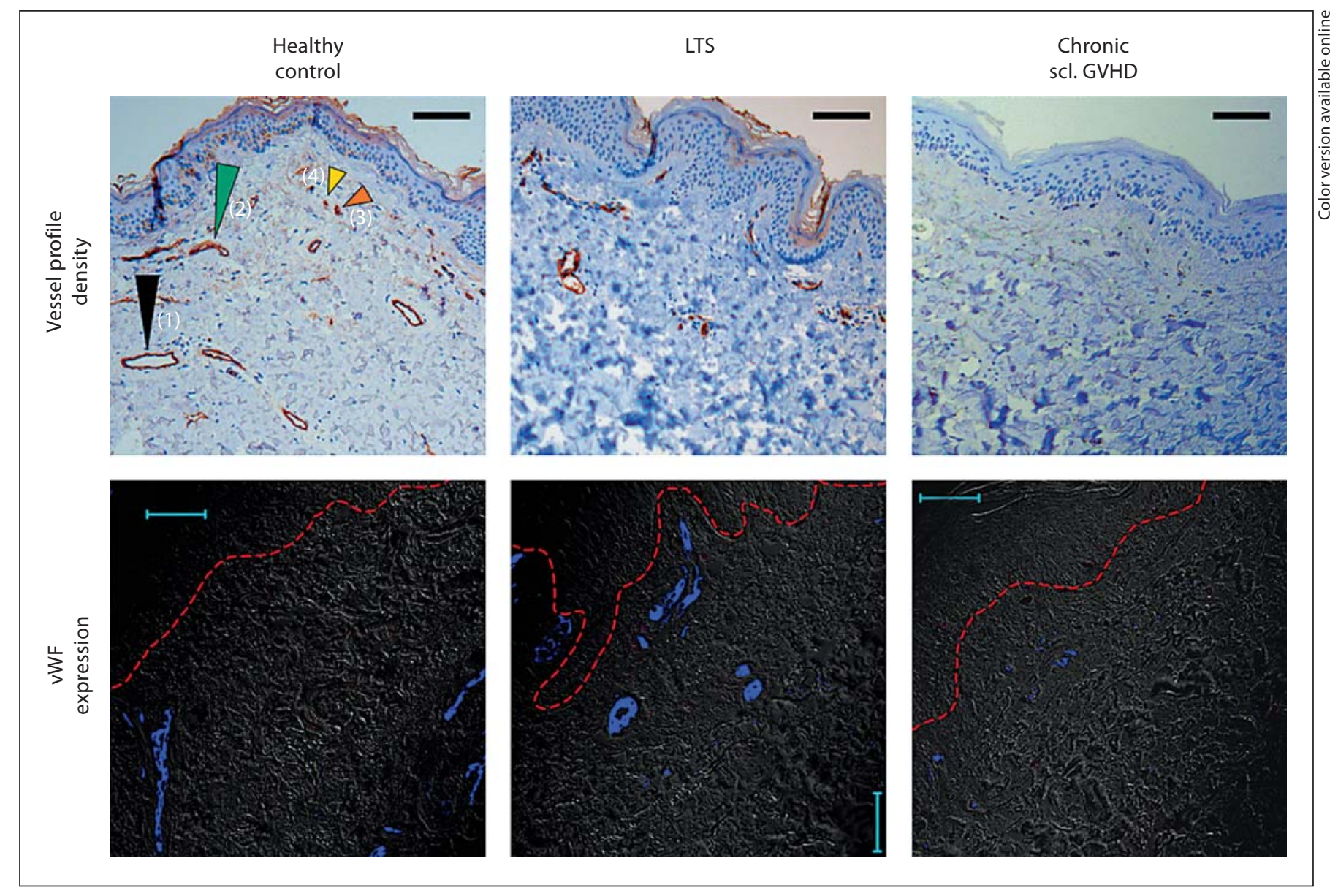

Fig. 2. Quantitative assessment of microvascular density in skin by two different methods. Blood vessels are detected by staining for vWF using IHC or IF. Upper panels: blood vessel detection in the superficial dermis by IHC using anti-vWF antibody and visualization with a horseradish peroxidase-conjugated secondary antibody and DAB EnVision kit (DAKO); lower panels: blood vessel detection in the superficial dermis by IF using anti-vWF antibody and a secondary, Cy5 fluorescent antibody (blue in the on- line version). Quantification is done by vessel profile count (upper panels) and by counting pixels that represent vWF expression (blue pixels in the online version, lower panels). The blood vessels are further categorized into four different size groups according to their diameter [upper panels: (1) black arrowhead: diameter $>20 \mu \mathrm{m}$, (2) green arrowhead: 10-20 $\mu \mathrm{m}$, (3) orange arrowhead: 0-10 $\mu \mathrm{m}$, (4) yellow arrowhead: no lumen or sprouts]. Bars = $50 \mu \mathrm{m}$; dotted red line: epidermis-dermis boundary. and nontransplanted healthy controls $(n=6)$. The anatomical findings of the superficial (fig. 1, upper row) and deep (fig. 1, lower row) dermis are shown with a highand low-power view, respectively. LTS and healthy controls hereby present similar morphological findings with respect to vessel count, vessel size and collageneous matrix. In contrast, chronic sclerotic GVHD reveals homogenization, enlargement and compactation of collagen fibers of the upper and lower dermis and rarification of vessels particularly in the upper dermal parts. Morphea, an idiopathic sclerotic skin disorder with prominent compactation and homogenization of collagen fibers pri- marily of deep dermal parts, discloses upper dermal matrix and vessel characteristics similar to those of LTS and healthy controls. Lichen sclerosus, a fibrosing skin disease with compactation and homogenization of collagen of the uppermost parts of the dermis, demonstrates prominent vessel rarification in the papillary dermis within a sclerotic matrix.

\section{Higher Interindividual Variability of Microvascular}

Density in LTS Compared to Healthy Controls

Two independent morphometric methods were used to assess microvascular density in the skin (for details, 


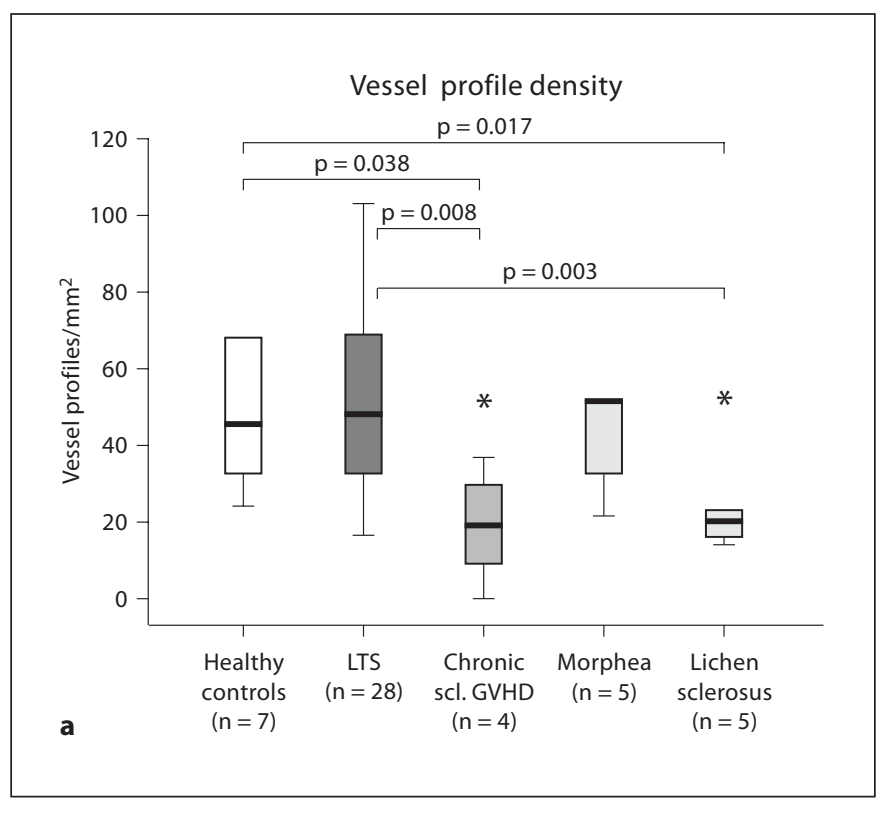

Fig. 3. Microvascular density in healthy controls, LTS, chronic sclerotic GVHD, morphea and lichen sclerosus. Vascular endothelial cells on paraffin sections were detected with anti-vWF antibody and visualized with a horseradish peroxidase-conjugated secondary antibody and DAB EnVision kit (DAKO) (a) or a Cy5conjugated secondary antibody (b). a The sum of all vessel profiles $/ \mathrm{mm}^{2}$ is equal in healthy controls, LTS and morphea. Patients with chronic sclerotic GVHD and lichen sclerosus show a significant reduction in vessel profile density compared to healthy controls $\left(^{*}\right)$. Results are shown as median vessel profile density \pm 1 st

see fig. 2 and Methods). Figure 3 indicates the microvascular density determined as counted absolute number of vessel profiles $/ \mathrm{mm}^{2}$ (fig. 3a) and as vWF-positive pixel number per total pixel number of the area analyzed (fig. 3b).

Equal vascular densities are assessed in LTS and healthy controls by both independent quantification methods (vessels $/ \mathrm{mm}^{2}: 48.3$ compared to $47.9, \mathrm{p}=0.947$; pixels: 1,190 compared to $1,138, \mathrm{p}=0.719$, Mann-Whitney $U$ test). This finding is independent of whether LTS previously experienced any GVHD or were currently classified as having chronic GVHD without skin involvement [vessels $/ \mathrm{mm}^{2}: 51.9$ (no GVHD) compared to 48.3 (any previous GVHD), $\mathrm{p}=0.872$; pixels: 902 (no GVHD) compared to 1,248 (any GVHD), $\mathrm{p}=0.478$, Mann-Whitney $\mathrm{U}$ test].

Compared to healthy controls (47.9 vessels $/ \mathrm{mm}^{2}$ and 1,138 pixels), an anticipated significant decrease in absolute vessel count was found in chronic sclerotic GVHD (19.3 vessels $/ \mathrm{mm}^{2}, \mathrm{p}=0.038 ; 678$ pixels, $\left.\mathrm{p}=0.018\right)$ and

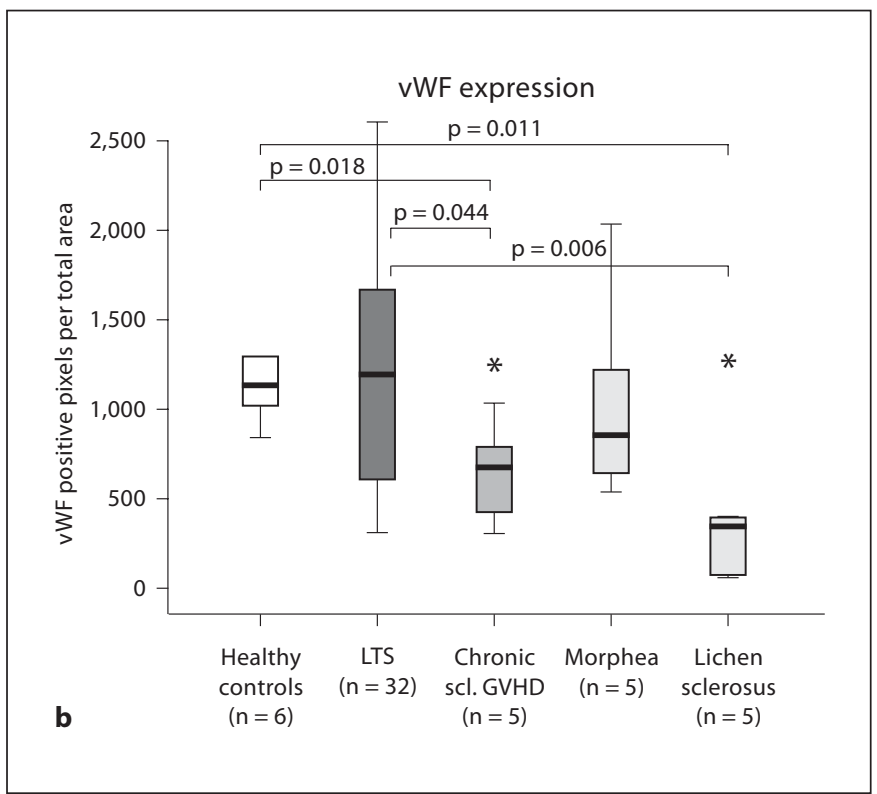

and 3rd quartiles $\left(\mathrm{p}_{\mathrm{LTS}}=0.947, \mathrm{p}_{\mathrm{cGVHD}}=0.038, \mathrm{p}_{\text {morphea }}=0.945\right.$, $\mathrm{p}_{\text {lichen sclerosus }}=0.017$, Mann-Whitney U test). $\mathbf{b}$ In silico measurements of vWF-positive areas (blue pixel numbers per area) confirm results shown in a. The sum of all vWF-positive pixels per area is equal in healthy controls, LTS and morphea. Patients with chronic sclerotic GVHD and lichen sclerosus show a significantly lower vWF pixel count than healthy controls $\left(^{*}\right)$. Results are shown as median pixel counts \pm 1 st and 3 rd quartiles $\left(\mathrm{p}_{\mathrm{LTS}}=\right.$ $0.719, \mathrm{p}_{\mathrm{cGVHD}}=0.018, \mathrm{p}_{\text {morphea }}=0.361, \mathrm{p}_{\text {lichen sclerosus }}=0.011$, Mann-Whitney U test).

lichen sclerosus (20.0 vessels $/ \mathrm{mm}^{2}, \mathrm{p}=0.017 ; 344$ pixels, $\mathrm{p}=0.011$ ). Also when compared to LTS, the two sclerotic disorders show a significant decrease in vessel count $(\mathrm{p}=$ $0.008)$ and vWF pixels per total area $(\mathrm{p}=0.044)$ for chronic sclerotic GVHD, as well as for lichen sclerosus ( $\mathrm{p}=$ 0.003 , vessels $/ \mathrm{mm}^{2} ; \mathrm{p}=0.006$, vWF pixels). In contrast, morphea does not demonstrate significant changes of vessel density ( 51.6 vessels $/ \mathrm{mm}^{2}, \mathrm{p}=0.945 ; 854 \mathrm{vWF}$ pixels, $\mathrm{p}=0.361$, Mann-Whitney $\mathrm{U}$ test). This is in accordance with the anatomical matrix changes particularly of the lower dermis sparing the upper parts (fig. 1).

However, LTS show a broad range of microvascular density as compared to healthy controls by both independent methods. This interindividual variability of microvascular densities within the LTS group was further explored by analyzing the four quartile groups (table 2; fig. 3a). The lowest subgroup of patients (1st quartile of data values) in fact shows a significant reduction in vessel density compared to healthy controls ( $p=0.003$ for vessels $/ \mathrm{mm}^{2}, \mathrm{p}=0.013$ for pixels, Mann-Whitney $\mathrm{U}$ test), 
Table 2. Quartile analysis of microvascular density in LTS

\begin{tabular}{llllllll}
\hline $\begin{array}{l}\text { LTS group } \\
\text { quartiles } \\
\text { (profile } \\
\text { numbers) }\end{array}$ & $\begin{array}{l}\text { TBI } \\
\text { (yes/no) }\end{array}$ & $\begin{array}{l}\text { Sex } \\
(\mathrm{m} / \mathrm{f})\end{array}$ & $\begin{array}{l}\text { Mean age at } \\
\text { transplantation } \\
\text { (range) }\end{array}$ & $\begin{array}{l}\text { Time interval } \\
\text { after HSCT } \\
\text { (mean years) }\end{array}$ & $\begin{array}{l}\text { Experienced } \\
\text { acute GVHD } \\
\text { grade } \\
\text { (I/II/III/IV) }\end{array}$ & $\begin{array}{l}\text { Experienced } \\
\text { chronic GVHD } \\
\text { grade (none/ } \\
\text { limited/extensive) }\end{array}$ & $\begin{array}{l}\text { Current chronic } \\
\text { GVHD grade } \\
\text { severe) }\end{array}$ \\
\hline $\begin{array}{l}1(17-28) \\
2(38-48)\end{array}$ & $5 / 1$ & $4 / 2$ & $27(18-34)$ & $17.4(11-25)$ & $2 / 1 / 3 / 0 / 0$ & $3 / 2 / 0$ & $6 / 0 / 0$ \\
$3(52-63)$ & $6 / 2$ & $6 / 4$ & $26(5-50)$ & $17.1(14-23)$ & $3 / 3 / 2 / 2 / 0$ & $4 / 4 / 2$ & $9 / 0 / 1$ \\
$4(75-103)$ & $8 / 1$ & $3 / 4$ & $31(20-44)$ & $16.8(12-20)$ & $2 / 2 / 2 / 1 / 0$ & $4 / 2 / 1$ & $5 / 2 / 0$ \\
\hline
\end{tabular}

The LTS group of GVHD patients has been divided into 4 subgroups according to the patient's vessel profile density; each group represents a quartile range. No correlation of the vessel density quartile groups with any of the observed parameters can be seen.

while the highest subgroup (4th quartile) demonstrates a significant increase in vessel density ( $p=0.032$ for vessels $/ \mathrm{mm}^{2}, \mathrm{p}=0.042$ for pixels, Mann-Whitney U test; data not shown). The 2nd and the 3rd quartiles are not different from the healthy controls. Importantly, when the 1 st and the 4 th quartiles were systematically investigated for parameters such as recipients' gender, age at transplantation, time interval after allogeneic HSCT, conditioning regimens with or without total body irradiation or previous or current status of GVHD (table 2), we did not find any association between one of these characteristics and vessel quantity.

Finally, we compared the two quantification methods (vessel counts versus pixel measurement) by correlating the two data sets. As we generated in parallel one total vessel number $/ \mathrm{mm}^{2}$ (IHC) and one pixel value per area for each patient sample, we compared each pair of corresponding values using the Spearman correlation analysis over all patient groups including healthy controls (a total of 50 pairs). Statistical analysis revealed a Spearman correlation coefficient of 0.406 and a $p$ value of 0.003 (data not shown), indicating a clear correlation between the two measurement methods.

\section{No Microvascular Remodeling in LTS and in Chronic Sclerotic GVHD}

Figure 4a indicates the absolute vessel counts stratified per size category of vWF-positive detected vessels $/ \mathrm{mm}^{2}$ by IHC in the upper dermis (150-200 mm measured from the basal membrane downwards). Irrespective of the size category of microvessels, LTS and healthy controls do not show any significant quantitative change in the vessel profile density (33.4 compared to 31.2 vessels $/ \mathrm{mm}^{2}, \mathrm{p}=$ 0.665). In contrast, significant decreases in the absolute number of vascular profiles in the smallest vessel catego- ry (sprouts without detectable lumen) was found in chronic sclerotic GVHD (11.3 vessels $/ \mathrm{mm}^{2}, \mathrm{p}=0.010$ ) and lichen sclerosus (12.5 vessels $\left./ \mathrm{mm}^{2}, \mathrm{p}=0.006\right)$ when compared to healthy controls $\left(33.4\right.$ vessels $/ \mathrm{mm}^{2}$ ). Analogously, when compared to LTS (31.2 vessels $/ \mathrm{mm}^{2}$ ), chronic sclerotic GVHD (11.3 vessels $/ \mathrm{mm}^{2}, \mathrm{p}=0.008$ ) as well as lichen sclerosus (12.5 vessels $/ \mathrm{mm}^{2}, \mathrm{p}=0.003$ ) showed a significant decrease in the absolute number of vascular profiles in the smallest vessel category. Furthermore, chronic sclerotic GVHD and lichen sclerosus demonstrate a relative but not significant increase in large microvessels $(>20 \mathrm{~mm})$.

By investigating the relative size distribution of microvessels per total vessel profile numbers (criteria for vascular remodeling), figure $4 \mathrm{~b}$ demonstrates that the very small vessels either with no or very thin lumens $(0-$ $10 \mathrm{~mm}$ ) are the most prevalent vascular structures in all groups relative to the total profile numbers $/ \mathrm{mm}^{2}$ and compared to healthy controls. Importantly, and here shown for all vessel size categories, LTS and healthy controls have a nearly identical vessel size distribution pattern.

Taken together, the terminal vascular bed in the uppermost skin of LTS is therefore almost identical to that of healthy controls in terms of the relative distribution of vessel size categories. Moreover, the relative size distribution of vessel profiles within one group (no lumen, $0-10$, $10-20,>20 \mathrm{~mm})$ did not show any significant changes $\left(\chi^{2}\right.$ test, $p$ values are indicated in fig. 4) compared to healthy controls in any of the four investigated groups. Particularly with respect to diseases with quantitative loss of microvessels such as chronic sclerotic GVHD and lichen sclerosus, there is no evidence that the microvascular loss would occur preferentially in one vessel size category. 


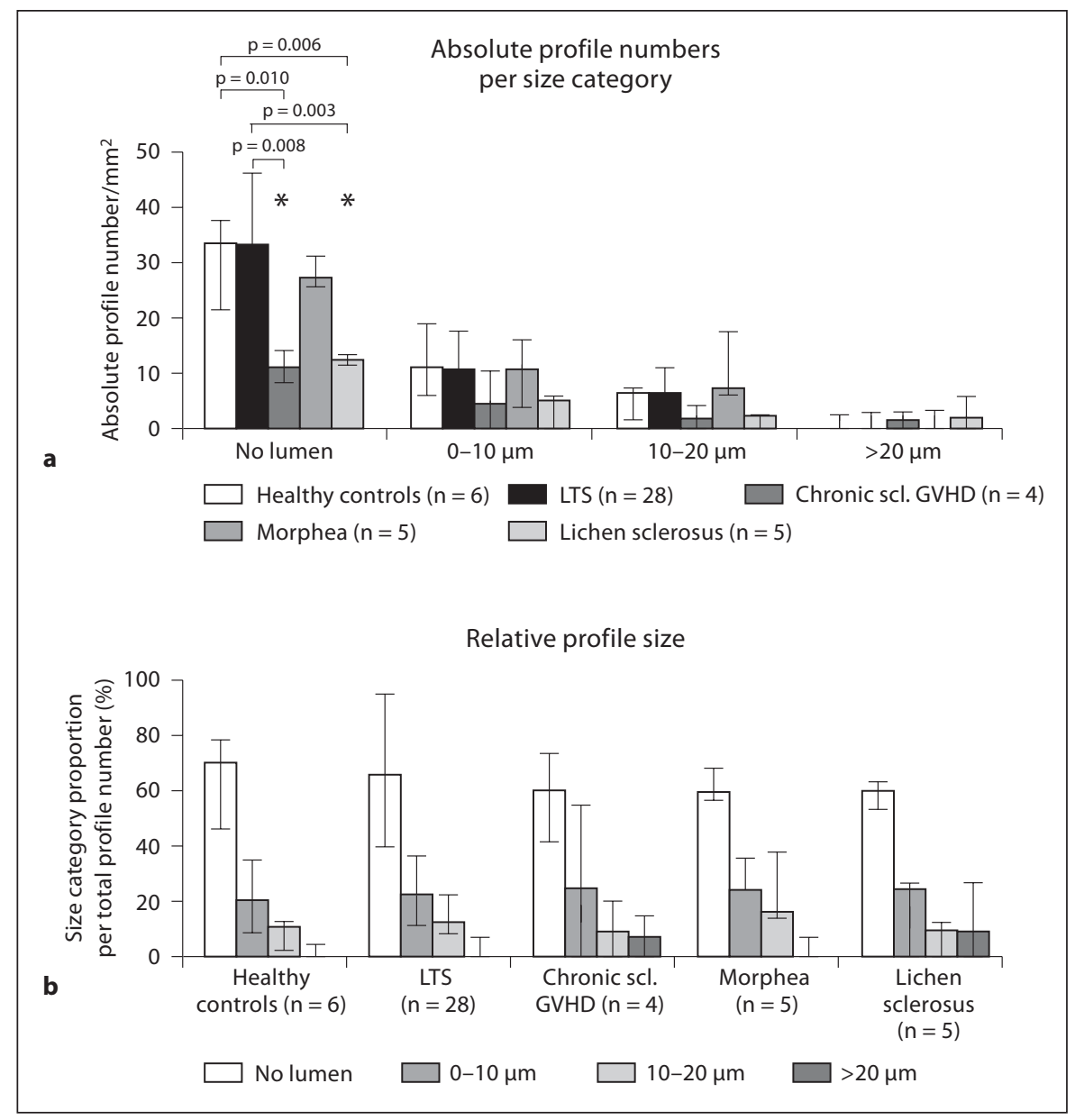

Fig. 4. Size distribution of cutaneous microvessels in healthy controls, LTS, chronic sclerotic GVHD, morphea and lichen sclerosus. Each vessel profile count was categorized according to its vessel diameter into the size categories 'no lumen', '0-10 $\mu \mathrm{m}$ ', ' $10-20$ $\mu \mathrm{m}$ ' and ' $>20 \mu \mathrm{m}$ '. Patient groups are shown in different grayscale bars. a Absolute vessel profile numbers per size categories. Results are shown as median vessel profile numbers \pm 1 st and 3 rd quartiles. Patients with chronic sclerotic GVHD and lichen sclerosus differ significantly from healthy controls in vessel profile numbers of the smallest category ('no lumen', $p=0.010$ and 0.006 , respectively, Mann-Whitney U test). Both patient groups show a tendency of reduction in numbers of vessels of an intermediate size, and a tendency to increase the numbers of the largest vessels

\section{Discussion}

In this observational study, we investigated the current status of the cutaneous microvasculature in a large homogeneous cohort of hematopoietic stem cell recipients more than 10 years after transplantation. Our primary aim was to capture all information about absolute number of vessels in skin (criteria for reconstitution) and
( $>20 \mu \mathrm{m})$; however, the tendency is not significant (all cases $\mathrm{p}>$ 0.05, Mann-Whitney U test). Patients with chronic sclerotic GVHD also differ significantly from LTS patients in the smallest vessel category $(p=0.008)$, indicating a normalization of the vessel system in LTS (restitutio ad integrum). b Vessel size distribution pattern per patient group. The vessel profile number from each size category (a) is divided by the total sum of all vessel profiles for each patient group. Results are shown as a percentage of total vessels (median percent \pm 1 st and 3 rd quartiles). All patient groups demonstrate comparable vessel size distribution patterns $\left(\mathrm{p}_{\mathrm{LTS}}=0.488, \mathrm{p}_{\mathrm{cGVHD}}=0.283, \mathrm{p}_{\text {morphea }}=0.395, \mathrm{p}_{\text {lichen sclerosus }}=\right.$ $0.186, \chi^{2}$ test). relative distribution of vessel size categories (criteria for remodeling) to objectify any subclinical long-term sequelae related to that therapy. Vascular structures were assessed according to immunohistochemical and IF reactivity for vWF (for details see fig. 2), a glycoprotein synthesized by vascular endothelial cells and stored in the Weibel-Palade bodies [15]. All recipients (LTS) revealed a high degree of homogeneity with respect to their condi- 
tioning and transplant procedures and did not show any clinical and histological signs of cutaneous GVHD at the time of follow-up and skin biopsy (table 1). Our study was part of a cooperative project related to the single-center prospective study in Basel entitled 'Altered immunity in long-term survivors after allogeneic hematopoietic stem cell transplantation as compared with their respective donors'.

Our main findings can be summarized as follows. First, LTS without clinical signs of chronic cutaneous GVHD and healthy controls demonstrate comparable morphological features with respect to dermal matrix and vascular structures (fig. 1) as compared to patients with chronic sclerotic GVHD and lichen sclerosus [14, 16]. Second, quantitative assessment of microvascular density by two independent methods (fig. $3 a, b$ ) shows identical absolute numbers of vessel profiles in LTS and healthy controls. Both measurement methods thereby correlate with a Spearman correlation coefficient of 0.406 (figure not shown). With respect to the increased interindividual variability in vessel density of LTS as compared to healthy controls, and based on the subsequent quartile group analysis of LTS (table 2), we were not able to identify any discriminating parameters within these subgroups such as sex and age at transplantation, conditioning regimens, years after transplantation, and, importantly, current or previous status of GVHD. This is particularly remarkable, as a significant blood vessel loss has been previously demonstrated in skin biopsies of allogeneic stem cell recipients with clinical features of chronic GVHD [14] and here confirmed by us (fig. 3). Third, LTS and healthy controls do essentially demonstrate an equal absolute vessel profile number per size category (fig. 4a) and a comparable relative vessel profile size distribution pattern (fig. 4b). These are crucial arguments against persistent vascular sequelae of skin of LTS more than 10 years after transplantation. Furthermore, we did not find any correlation of the serum vWF of these LTS (data not shown) to any of the clinical parameters listed in table 2 and their respective vascular density. It finally could be hypothesized that beyond previous occurrence or absence of GVHD, peritransplant procedures and diseases such as viral infections do not have a sustained impact on the terminal vascular bed in skin of LTS.

Human microvasculature in normal skin and pathological conditions have previously been visualized in detail using laser Doppler flowmetry highlighting the microanatomical organization of the upper and lower horizontal vascular plexus and the connecting vessels [17-19].
Past investigations elucidated that various immune-mediated fibrosing skin diseases result in similar modifications of vascular structures, cellular and molecular events as well as matrix changes. Vertically oriented capillary loops originating from and terminating in the upper vascular plexus were found to be transpositioned in lichen sclerosus from vertical to horizontal orientation toward the basal membrane zone [16]. Induction of sclerosis in fibrosing skin diseases such as chronic sclerotic GVHD, morphea and lichen sclerosus has been demonstrated to be critically mediated by TGF- $\beta$-induced activation of fibroblasts with subsequent collagen production and the simultaneous stimulation of tissue inactivators for metalloproteinases and increases in platelet-derived growth factor- $\alpha$ receptor expression in fibroblasts and endothelial endothelin-1 synthesis [20,21].

Our findings in LTS address further vascular aspects to the enhanced risk of premature cardiovascular disease recently demonstrated in 265 recipients of allogeneic hematopoietic stem cell transplants with a median followup of 9 years [22]. Though these late vascular events have not been observed in a patient cohort comparable to our homogeneous group of LTS, a relevant number of registered arterial episodes in these patients such as stroke, coronary heart disease, or peripheral arterial disease have been observed many years after transplantation with well-defined risk factors for endothelial damage such total body irradiation [23] or GVHD [14]. Essentially, the terminal vascular bed in skin comprises mainly capillaries, postcapillary venules, and few arterioles, but lack larger arteries. Hypothetically, micro- and macrovascular structures biologically behave differently after acute and/or chronic damage and might have unequal capabilities to recover.

In summary, in this study we demonstrated the complete remodeling and partial reconstitution of the terminal vascular bed in skin of a homogeneous cohort of LTS more than 10 years after allogeneic HSCT and irrespective of the former occurrence of GVHD involving skin. It can be hypothesized that peritransplant procedures such as total body irradiation, chemotherapy, GVHD, drug reactions or infections do not have a sustained impact on the ability of the skin to recover a complete microvasculare network many years after allogeneic HSCT.

\section{Acknowledgments}

We would like to thank Ms Denise Dubler for her skillful technical assistance. This work was funded by the Department of Dermatology, University Hospital Basel. 


\section{References}

1 Sullivan KM: Graft-versus-host disease; in Forman SJ, Blume KG, Thomas ED (eds): Bone Marrow Transplantation. Cambridge, Blackwell Scientific, 1994, pp 339-362.

-2 Storek J, Gooley T, Witherspoon RP, Sullivan $\mathrm{KM}$, Storb R: Infectious morbidity in longterm survivors of allogeneic marrow transplantation is associated with low CD4 T cell counts. Am J Hematol 1997;54:131-138.

- 3 Socie G, Stone JV, Wingard JR, Weisdorf D, Henslee-Downey PJ, Bredeson C, Cahn JY, Passweg JR, Rowlings PA, Schouten HC, Kolb HJ, Klein JP: Long-term survival and late deaths after allogeneic bone marrow transplantation. Late Effects Working Committee of the International Bone Marrow Transplant Registry. N Engl J Med 1999;341: $14-21$

-4 Mielcarek M, Martin PJ, Leisenring W, Flowers ME, Maloney DG, Sandmaier BM, Maris MB, Storb R: Graft-versus-host disease after nonmyeloablative versus conventional hematopoietic stem cell transplantation. Blood 2003;102:756-762.

$\checkmark 5$ Deeg HJ, Spitzer TR, Cottler-Fox M, Cahill R, Pickle LW: Conditioning-related toxicity and acute graft-versus-host disease in patients given methotrexate/cyclosporine prophylaxis. Bone Marrow Transplant 1991;7: 193-198.

6 Weisdorf DJ: Chronic graft-versus-host disease: where is promise for the future? Leukemia 2005; 19:1532-1535.

7 Hausermann P, Walter RB, Halter J, Biedermann BC, Tichelli A, Itin P, Gratwohl A: Cutaneous graft-versus-host disease: a guide for the dermatologist. Dermatology 2008; 216:287-304.
8 Ferrara JLM, Cooke KR, Teshima T: The pathophysiology of graft-versus-host disease; in Ferrara JLM, Cooke KR, Deeg HJ (eds): Graft-versus-Host Disease. New York, Dekker, 2005, pp 1-34.

9 Hofmeister CC, Quinn A, Cooke KR, Stiff P, Nickoloff B, Ferrara JL: Graft-versus-host disease of the skin: life and death on the epidermal edge. Biol Blood Marrow Transplant 2004;10:366-372.

10 Cutler C, Antin JH: Chronic graft-versushost disease. Curr Opin Oncol 2006;18:126131.

11 Sullivan KM: Graft-versus-host disease; in Blume KG, Forman SJ, Appelbaum FR (eds): Thomas' Hematopoietic Cell Transplantation, ed 3. Malden, Blackwell Publishing, 2004, pp 635-664

12 Lawley TJ, Peck GL, Moutsopoulos HM Gratwohl AA, Deisseroth AB: Scleroderma, Sjogren-like syndrome, and chronic graftversus-host disease. Ann Intern Med 1977; 87:707-709.

13 Gratwhol AA, Moutsopoulos HM, Chused TM, Akizuki M, Wolf RO, Sweet JB, Deisseroth AB: Sjogren-type syndrome after allogeneic bone-marrow transplantation. Ann Intern Med 1977;87:703-706.

14 Biedermann BC, Sahner S, Gregor M, Tsakiris DA, Jeanneret C, Pober JS, Gratwohl A: Endothelial injury mediated by cytotoxic $\mathrm{T}$ lymphocytes and loss of microvessels in chronic graft versus host disease. Lancet 2002;359:2078-2083.

15 Blann AD, Taberner DA: A reliable marker of endothelial cell dysfunction: does it exist? Br J Haematol 1995;90:244-248.
16 Kowalewski C, Kozlowska A, Gorska M, Wozniak K, Krajewski M, Blaszczyk M, Jablonska S: Alterations of basement membrane zone and cutaneous microvasculature in morphea and extragenital lichen sclerosus. Am J Dermatopathol 2005;27:489-496.

17 Braverman IM: Ultrastructure and organization of the cutaneous microvasculature in normal and pathologic states. J Invest Dermatol 1989;93:2S-9S

18 Braverman IM: The cutaneous microcirculation: ultrastructure and microanatomical organization. Microcirculation 1997;4:329340 .

19 Braverman IM: The cutaneous microcirculation. J Investig Dermatol Symp Proc 2000; 5:3-9.

20 Smith EA, LeRoy EC: A possible role for transforming growth factor-beta in systemic sclerosis. J Invest Dermatol 1990;95:125S$127 \mathrm{~S}$.

21 Sapadin AN, Esser AC, Fleischmajer R: Immunopathogenesis of scleroderma - Evolving concepts. Mt Sinai J Med 2001;68:233242 .

22 Tichelli A, Bucher C, Rovo A, Stussi G, Stern M, Paulussen M, Halter J, Meyer-Monard S, Heim D, Tsakiris DA, Biedermann B, Passweg JR, Gratwohl A: Premature cardiovascular disease after allogeneic hematopoietic stem-cell transplantation. Blood 2007;110: 3463-3471.

23 Basavaraju SR, Easterly CE: Pathophysiological effects of radiation on atherosclerosis development and progression, and the incidence of cardiovascular complications. Med Phys 2002;29:2391-2403. 